\title{
Investigação de Linfonodos em Pacientes em Seguimento por Carcinoma Diferenciado de Tiróide
}

\begin{abstract}
RESUMO
Recorrências regionais dos carcinomas diferenciados de tiróide (CDT) são representadas por linfonodos cervicais em $60-75 \%$ dos casos. Com a introdução da ultra-sonografia cervical (USC) no seguimento dos pacientes com carcinoma papilífero de tiróide (CPT) tornou-se freqüente o encontro de pequenos linfonodos (LNs) cervicais. Porém, apesar da USC apresentar alta sensibilidade, o estudo citológico obtido por punção aspirativa (PAAF), e nos últimos anos, a dosagem da tiroglobulina ( $\mathrm{Tg}$ ) no lavado da agulha da PAAF (Tg-PAAF), vêm assumindo papel importante no diagnóstico de LNs cervicais. O objetivo deste estudo é revisar a importância do diagnóstico precoce das metástases em linfonodos cervicais no seguimento dos pacientes com carcinoma de tiróide. (Arq Bras Endocrinol Metab 2007;51/5:813-817)
\end{abstract}

Descritores: Câncer de tiróide; Punção aspirativa com agulha fina; Tiroglobulina; Ultra-sonografia; Metástases em linfonodos

\begin{abstract}
Cervical Lymph Nodes Metastases in Patients With Differentiated Thyroid Cancer.

Loco-regional recurrences of the differentiated thyroid cancer have been reported to be located in cervical lymph nodes in $60-75 \%$ of cases. The widespread use of neck ultrasonography (US) during the follow-up of patients with papillary thyroid carcinoma (PTC) has led to the discovery of small cervical lymph nodes (LN). Although US has a high sensitivity for diagnosing LN, fine needle aspiration biopsy (FNA) and measurement of thyroglobulin in fine needle aspirates (FNA-Tg) have proven to be invaluable tools. The aim of this paper is to review the importance of the early diagnosis of lymph node metastases in the follow-up of patients with differentiated thyroid cancer. (Arq Bras Endocrinol Metab 2007;51/5:813-817)
\end{abstract}

Keywords: Thyroid cancer; Fine needle aspiration biopsy; Thyroglobulin; Ultrasound; Lymph nodes metastases

\footnotetext{
A presença de metástases em Linfonodos cervicais, em 30-75\% dos A pacientes, e de extensão extra-tireoidiana do tumor ao diagnóstico, tem sido identificada como fator de risco independente para recorrência da doença, porém ainda é discutível seu papel na sobrevida dos pacientes (16). Não está claro se cirurgias radicais com esvaziamento cervical bilateral resultam em aumento da taxa de sobrevida ou se a extensão de tais cirurgias aumentaria as complicações pós-operatórias (7-10). Por outro lado, tratamentos incompletos levam ao diagnóstico tardio de metástases cervicais interferindo no prognóstico e na morbidade dos pacientes (1-2).

Após a cirurgia, 5 a $20 \%$ dos pacientes com carcinoma diferenciado de tiróide apresentam persistência ou recorrência local ou regional da doença $(3-5)$.

A persistência de doença em leito tireoidiano ou em linfonodos geralmente associa-se a tratamento inicial incompleto (lobectomia ou tireoidectomia subtotal), variantes histológicas mais agressivas (carcinoma papilifero esclerosante difuso, células altas e outras), impossibilidade de remoção completa de alguns tumores (seja no leito tireoidiano ou por invasão em tecidos moles) ou pela falta de remoção de linfonodos metastáticos $(4,6,11,12)$. Por
}

\section{revisão}

Rosa paula M. Biscolla

Divisão de Endocrinologia, Escola Paulista de Medicina UNIFESP/EPM, São Paulo, SP.
Recebido em 12/02/07

Aceito em 15/02/07 
outro lado, recorrência é definida como evidência de doença de 6 a 12 meses após o paciente ser considerado livre de doença; das recorrências cervicais, 20\% ocorrem em leito tireoidiano, 60-75\% em linfonodos e 5\% em traquéia e músculos $(3,6,12-14)$.

\section{PRESENCCA DE LINFONODOS NOS PACIENTES EM SEGUIMENTO POR CDT}

A presença de linfonodos cervicais nos pacientes em seguimento por CDT é freqüente e, na maioria das vezes, não representa metástases. A maioria dos linfonodos cervicais é representada por linfoadenites reacionais, porém é importante incluir no diagnóstico diferencial as doenças granulomatosas (tuberculose, sarcoidose, histoplasmose), linfoma Hodgkin e metástases de tumores extra-tireoidianos (como pulmão, mama, esôfago, laringe e outros) (15-22).

Alguns estudos mostram que a possibilidade de um linfonodo cervical, em pacientes em seguimento por CDT submetido a tratamento inicial com tireoidectomia total e radioiodo, ser metástase varia de $9-56 \%(15,16,18,19,21,22)$. A grande variação encontrada ocorre devido ao pequeno número de pacientes em alguns estudos $(15,18)$ e à inespecificidade do linfonodo puncionado $(15,16,18,19)$ (tabela 1). Portanto, é fundamental que ao encontro de linfonodos cervicais nos pacientes em seguimento por CDT as características ultra-sonográficas do linfonodos sejam avaliadas cuidadosamente.

Atualmente, é consenso que os pacientes com AcTg negativos, submetidos a tireoidectomia total e ablação do tecido remanescente com radioiodo, com valores indetectáveis de sTg após teste de estímulo com TSH recombinante ou em hipotireoidismo, devam ser seguidos com dosagens de sTg anuais sob terapia com LT4 e com USC (11,23-25). Nos últimos anos, a USC assumiu papel fundamental no seguimento dos pacientes com carcinoma diferenciado de tiróide (CDT), principalmente, no carcinoma papilífero de tiróide, e deve ser realizada de rotina em todos os pacientes, independente- mente do risco inicial, sendo considerado o exame mais sensível para o diagnóstico das recorrências e metástases cervicais $(11,12,23-26)$.

\section{CARACTERÍSTICAS ULTRA-SONOGRÁFICAS DOS LINFONODOS CERVICAIS}

$\mathrm{Na}$ literatura, características ultra-sonográficas de linfonodos reacionais e malignos já estão bem estabelecidas $(22,27-31)$. A presença de linfonodos ovalados ou alongados (razão eixo curto/eixo longo $<0,5$ ou 0,7 ), com hilo hiperecogênico central, de ecotextura hipoecogênica uniforme e localizados no $1 / 3$ superior do pescoço (regiões submentoniana, submandibular e jugular superior) é característica de linfonodos reacionais $(22,28-31)$. Por outro lado, linfonodos arredondados (razão eixo curto/eixo longo $>0,5$ ou 0,7 ), com estrutura heterogênea (geralmente hipoecogênico com perda do hilo e algumas vezes com presença de calcificações e/ou degenerações císticas), localizados no $1 / 3$ inferior do pescoço (níveis IV, V e VI) são fortemente suspeitos de metástases de CDT $(22,29-31)$. Calcificações finas podem ser encontradas em metástases de carcinoma papilífero em 50-69\% dos casos; porém, na presença de calcificações grosseiras, o diagnóstico diferencial de tuberculose deve ser excluído $(28,32,33)$.

\section{AVALIAÇÃO CITOLÓGICA DOS LINFONODOS VISUALIZADOS À USC EM PACIENTES COM CDT}

Embora a sensibilidade da USC em detectar massas ocultas e linfonodos cervicais não palpáveis esteja bem documentada, a capacidade de diferenciar linfonodos "benignos" (inflamatórios ou reacionais) de linfonodos "malignos" (metastáticos) é baixa $(27,30)$. Após a detecção de linfonodos "suspeitos" à USC, é necessária a realização de estudo citológico obtido através da punção aspirativa por agulha fina (PAAF) $(27,30,34,35)$. O estudo citológico obtido da PAAF de um linfonodo suspeito à USC

Tabela 1. Presença de metástases em linfonodos de pacientes em seguimento por carcinoma de tiróide.

\begin{tabular}{lccc}
\hline Autor & Pacs & Característica Linfonodo Puncionado & Metástases Ca tiróide* \\
Pacini e cols., 1992 & 23 & Aumentado & $10(43 \%)$ \\
Frasoldati e cols., 1999 & 47 & Aumentado & $9(19 \%)$ \\
Cignarelli e cols., 2003 & 16 & $>10 \mathrm{~mm}$ & $9(56 \%)$ \\
Baskin e cols., 2004 & 74 & $5 \mathrm{~mm}$ sem hilo central & $7(9 \%)$ \\
Boi e cols., 2006 & 41 & Ovalado, com calcif. vascul difusa & $23(56 \%)$ \\
Biscolla e cols., 2007 & 32 & Ovalado, hipoecogênico, sem hilo central & $11(34 \%)$ \\
\hline
\end{tabular}

* Diagnóstico com PAAF e Tg-PAAF e confirmação com exame histológico. 
apresenta valor fundamental, principalmente nos pacientes com dosagens de tireoglobulina sérica $(\mathrm{sTg})$ indetectáveis em vigência de TSH suprimido (20\% dos pacientes) (36) e naqueles com metástases não iodo-captantes (20-40\% dos pacientes) $(37,38)$.

Os resultados mais freqüientemente encontrados com a realização da PAAF são: "linfonodo reacional", quando a citologia mostra presença de linfócitos em diferentes estágios de maturação; "linfonodo metastático", quando à citologia encontram-se células foliculares em substituição à população linfocitária; ou "não diagnóstica", quando o estudo citológico é prejudicado pela insuficiência de material obtido. Menos freqüentemente, podemos encontrar achados compatíveis com doenças granulomatosas (tuberculose, sarcoidose), com neoplasias como linfomas ou metástases de outros carcinomas (laringe, pulmão ou mama) $(15,18)$. Em 32 pacientes com carcinoma papilífero tratados com tireoidectomia total e ${ }^{131} \mathrm{I}$, em seguimento no ambulatório de tireóide da UNIFESP, os resultados da PAAF, realizada em 44 linfonodos visualizados ao USC, foram "linfonodos reacionais" em 65\%, "linfonodos metastáticos" em $29 \%$ e material "não diagnóstico" em 6\% (22). Este último dado está de acordo com a literatura, que refere resultado não diagnóstico em aproximadamente $8 \%$ das citologias $(34,35,39)$.

\section{DOSAGEM DA TIREOGLOBULINA OBTIDA DO LAVADO DA AGULHA UTILIZADA NA REALIZAÇÃO DA PAAF (Tg-PAAF)}

Nos últimos anos, a dosagem da $\mathrm{Tg}$, obtida do lavado da agulha utilizada na realização da PAAF (Tg-PAAF) em linfonodos suspeitos tem sido proposta a fim de se diferenciar linfonodos "reacionais" (encontrados habitualmente à USC de rotina) de linfonodos “metastáticos”. A partir de 1992, alguns grupos começaram a dosar a Tg no lavado da agulha utilizada para a punção de linfonodo suspeito e, desde então, vários estudos têm mostrado que altos níveis de Tg-PAAF indicam a presença de metástase de CDT, enquanto que níveis indetectáveis de Tg-PAAF afastam essa possibilidade (15-22).

Pacini e cols. foram os primeiros a demonstrar níveis elevados de Tg-PAAF em linfonodos com metástases de carcinoma de tiróide, enquanto que valores indetectáveis indicavam linfoadenopatia inflamatória ou de origem não tiroidiana. Nesses pacientes, a sensibilidade da $\mathrm{Tg}$ PAAF foi $100 \%$, enquanto que a sensibilidade da citologia foi $85,7 \%$ (15).

Alguns anos depois, Frasodalti e cols. mostraram que a combinação da citologia com a Tg PAAF permitiu a detecção de linfonodos metastáticos em número maior que cada técnica individualmente, sendo que a realização conjunta da PAAF e da $\mathrm{Tg}$ PAAF apresentava sensibilidade maior no grupo de pacientes com linfonodos cervicais do que a realização da PCI ou a dosagem de sTg (16). O mesmo grupo demonstrou, recentemente, em pacientes com recorrência cervical de carcinoma diferenciado de tireóide, que a associação da dosagem de Tg PAAF ao exame citológico aumentou a sensibilidade do método de $84,8 \%$ para $95,6 \%(17)$.

A partir de 2004, vários autores demonstraram que linfonodos metastáticos apresentam níveis elevados de Tg-PAAF, enquanto que linfonodos inflamatórios apresentam níveis baixos de Tg-PAAF (19-21).

Nos pacientes em acompanhamento no ambulatório de tireóide da UNIFESP, a realização da citologia permitiu o diagnóstico de linfonodo reacional ou metastático em 95\% dos pacientes com sensibilidade de $86 \%$, especificidade de $100 \%$ e valor preditivo positivo e negativo de $100 \%$. Com o acréscimo da dosagem da TgPAAF à citologia, a sensibilidade e a especificidade passaram para $100 \%$, já que 2 pacientes apresentavam dosagens altas de Tg-PAAF com obtenção de material insuficiente para estudo citológico (22).

Portanto, os trabalhos da literatura realizados com pacientes tratados com tireoidectomia e radioiodo são concordantes em que, na ausência de tecido tireoidiano, os linfonodos reacionais apresentam níveis indetectáveis ou muito baixos de Tg-PAAF $(15,18,19)$. Esses valores muito baixos referidos em alguns artigos, como dosagens $<5 \mathrm{ng} / \mathrm{dl}$, podem ocorrer devido a interferentes (noise) nos ensaios (15). Valores muito baixos de Tg-PAAF não devem ser considerados como suspeitos de metástases, e acompanhamento seriado dos linfonodos com ultra-som e posterior repunção, se necessária, é a melhor conduta para nestes casos.

Por outro lado, Mariotti e col. alertam para a dosagem de Tg-PAAF em linfonodos de pacientes não tireoidectomizados. Nesses pacientes, os autores estabeleceram um cut-off para que a dosagem de Tg-PAAF fosse discriminatória entre linfonodos reacionais ou metastáticos (2l). É importante lembrar que pacientes que apresentam níveis de sTg elevados, seja por apresentaram tecido tireoidiano (pacientes não submetidos à tireoidectomia total) ou pacientes que apresentam níveis de $s T g$ elevados devido à presença de metástases a distância, podem ter no momento da coleta da TgPAAF sua amostra "contaminada" com sangue, o que poderia levar a resultados falso-positivos da Tg-PAAF. Além disso, durante a realização da PAAF de linfonodos por profissional não experiente, em pacientes com 
tireóide, pode haver contato da agulha com tecido tireoidiano, o que levaria à contaminação do material com altas quantidades de $\mathrm{Tg}$, resultando em dosagens elevadas de Tg-PAAF. Portanto, novos estudos são necessários para melhor avaliação do comportamento da Tg-PAAF em linfonodos de pacientes não tireoidectomizados.

\section{CONCLUSŌES}

A imagem à USC é importante para diferenciar linfonodos que devam ser puncionados de linfonodos que devam ser seguidos com exames de imagem de rotina.

$\mathrm{Na}$ presença de linfonodos cervicais suspeitos à USC, em pacientes em seguimento por carcinoma diferenciado de tiróide, principalmente pacientes com carcinoma papilífero, tratados com tireoidectomia total, deve-se realizar estudo citológico do linfonodo e dosagem de Tg-PAAF. A dosagem da Tg-PAAF deve ser sempre comparada com a dosagem da sTg e atenção às situações que possam resultar em resultados falso-positivos devem ser avaliadas em cada paciente.

Novos estudos são necessários para esclarecer sobre a dosagem da Tg-PAAF em pacientes não tireoidectomizados.

\section{REFERÊNCIAS}

1. Leboulleux S, Rubino C, Baudin E, Caillou B, Hartl DM, Bidart $\mathrm{JM}$, et al. Prognostic factors of persistent or recurrent disease of papillary thyroid carcinoma with neck lymph node metastases and/or tumor extension beyond the thyroid capsule at initial diagnosis. J Clin Endocrinol Metab 2005; 90(10):5723-9.

2. Rouxel A, Hejblum G, Bernier MO, Boelle PY, Ménégaux F, Mansour G, et al. Prognostic factors associated with the survival of patients developing loco-regional recurrences of differentiated thyroid carcinomas. J Clin Endocrinol Metab 2004;90(11):5362-8.

3. Mazzaferri EL, Kloos R. Current Approaches to primary therapy for papillary and follicular thyroid cancer. J Clin Endocrinol Metab 2001;86:1447-63.

4. Schlumberger MJ. Medical progress: papillary and follicular thyroid carcinoma. N Eng J Med 1998;338:297-306.

5. Sherman SI. Thyroid carcinoma. Lancet 2003;361:501-11.

6. De Groot LJ, Kaplan EL, McCormick M, Strauss FH. Natural history, treatment, and course of papillary thyroid carcinoma. J Clin Endocrinol Metab 1990;71:414-24.

7. Grebe S, Hay ID. Thyroid cancer nodal metastases: biologic significance and therapeutic considerations. Surg Oncol Clin N Am 1996:5:43-53.

8. Attie JN. Modified neck dissection in the treatment of thyroid cancer: a safe procedure. Eur J Cancer Clin Oncol 1988;24:315-21.

9. Noguchi S, Mizukami N. The value of lymph node dissection in patients with differentiated thyroid cancer. Surg Clin North Am 1987;67:251-61.

10. Simon D, Goretzki PE, WitteJ, Foher HD. Incidence of regional recurrence guiding radicality in differentiated thyroid carcinoma. World J Surg 1996;20:860-6.
11. Cooper DS, Doherty GM, Haugen BR, Kloos RT, Lee SL, Mandel SJ, et al. Management guidelines for patients with thyroid nodules and differentiated thyroid cancer. Thyroid 2006; $16: 1-33$

12. Schlumberger M, Pacini F. Papillary and follicular thyroid carcinoma. In: Thyroid Tumors. 2nd ed. France: Nucléon, 2003. pp. 111-81.

13. Maciel RMB, Biscolla RPM. Carcinoma papilífero e folicular da tiróide. In: Coronho V, Petroianu A, Santana EM, Pimenta LG. Tratado de Endocrinologia e Cirurgia Endócrina. 1a ed. São Paulo: Guanabara Koogan, 2001. pp. 530-42.

14. Mazzaferri EL, Jhiang SM. Long-term impact of initial surgical and medical therapy on papillary and follicular thyroid cancer. AM J Med 1994;97:418-28.

15. Pacini F, Fugazzola L, Lippi F, Ceccarelli C, Centoni R, Miccoli $P$, et al. Detection of thyroglobulin in fine needle aspirates of nonthyroidal neck masses: a clue to the diagnosis of metastatic differentiated thyroid cancer. J Clin Endocrinol Metab 1992; $74: 1401-4$.

16. Frasoldati A, Toschi E, Zini M, Flora M, Caroggio A, Dotti C, et al. Role of thyroglobulin measurement in fine-needle aspiration biopsies of cervical lymph nodes in patients with differentiated thyroid cancer. Thyroid 1999:9:105-11.

17. Frasoldati A, Pesenti M, Gallo M, Caroggio A, Salvo D, Valcavi $R$. Diagnosis of neck recurrences in patients with differentiated thyroid carcinoma. Cancer 2003:97:90-6.

18. Cignarelli M, Ambrosi A, Marino A, Lamacchia O, Campo M, Picca $G$, et al. Diagnostic utility of thyroglobulin detection in fine-needle aspiration of cervical cystic metastatic lymph nodes from papillary thyroid cancer with negative cytology. Thyroid 2003;12:1163-7.

19. Baskin HJ. Detection of recurrent papillary thyroid carcinoma by thyroglobulin assessment in the needle washout after fine-needle aspiration of suspicious lymph nodes. Thyroid 2004; 14:959-63.

20. Uruno T, Miyauchi A, Shimizu K, Tomoda C, Takamura Y, Ito $Y$, et al. Usefulness of thyroglobulin measurement in fineneedle aspiration biopsy specimens for diagnosing cervical lymph node metastasis in patients with papillary thyroid cancer. World J Surg 2005;29:483-5.

21. Boi F, Baghino G, Atzeni F, Lai ML, Faa G, Mariotti S. The diagnostic value for differentiated thyroid carcinoma metastases of thyroglobulin $(\mathrm{Tg})$ measurement in washout fluid from fine needle aspiration biopsy of neck lymph nodes is maintained in the presence of circulating anti-Tg antibodies. J Clin Endocrinol Metab 2006;91:1364-9.

22. Biscolla RP, Ikejiri E, Mamone MC, Nakabashi C, Andrade VP, Kasamatsu TS, et al. Diagnóstico de metástases de carcinoma papilífero de tiróide através da dosagem de tiroglobulina no líquido obtido da lavagem da agulha utilizada na punção aspirativa. Arq Bras Endocrinol Metab 2007;51(3):419-25.

23. Mazzaferri EL, Robbins RJ, Spencer CA, Braverman LE, Pacini $F$, Wartofsky $L$, et al. Consensus report of the role of serum thyroglobulin as a monitoring method for low-risk patients with papillary thyroid carcinoma. J Clin Endocrinol Metab 2003;88:1433-41.

24. Schlumberger M, Berg G, Cohen O, Duntas L, Jamar F, Jarzab B, et al. Follow up of low-risk patients with differentiated thyroid carcinoma: a European perspective. Eur J Endocrinol 2004;150:105-12.

25. Pacini $F$, Schlumberger $M$, Dralle $H$, Elisei $R$, Smit JW Wiersinga W, et al. European consensus for the management of patients with differentiated thyroid carcinoma of the follicular epithelium. Eur J Endocrinol 2006;154(6):787-803.

26. Pacini F, Molinaro E, Castagna MG, Agate L, Elisei R, Ceccarelli $\mathrm{C}$, et al. Recombinant human thyrotropin-stimulated serum thyroglobulin combined with neck ultrasonography has the highest sensitivity in monitoring differentiated thyroid carcinoma. J Clin Endocrinol Metab 2003;88:366873.

27. Dessler A, Rapparport Y, Blank A, Marmor S, Weiss J, Graif M. Cystic appearance of cervical lymph nodes is characteristic of metastatic papillary thyroid carcinoma. J Clin Ultrasound 2003;1:21-5. 
28. Rosário PW, Faria S, Bicalho L, Alves MF, Borges M, Purisch $S$, et al. Ultrasonographic differentiation between metastatic and benign lymph nodes in patients with papillary thyroid carcinoma. J Ultrasound Med 2005;24:1385-9.

29. Kuna S. Ultrasonographic differentiation of benign from malignant neck lymphadenopathy in thyroid cancer. J Ultrasound Med 2006;25:1531-7.

30. Sutton RT, Reading CC, Charboneau JW, James EM, Grant CS, Hay ID. US-guided biopsy of neck masses in postoperative management of patients with thyroid cancer. Radiology 1988; 168:769-72.

31. Antonelli A, Miccoli P, Ferdeghini M, Di Coscio G, Alberti B lacconi $P$, et al. Role of neck ultrasonography in the follow-up of patients operated on for thyroid cancer. Thyroid 1995;5(1):25-8

32. Ahuja A, Ying M, King W, Metreweli C. A practical approach to ultrasound of cervical lymph nodes. J Laryngol Otol 1997; $111: 245-56$.

33. Ahuja AT, Chow L, Chick W, King W, Metreweli C. Metastatic cervical nodes in papillary carcinoma of the thyroid: ultrasound and histological correlation. Clin Radiol 1995;50:22931.

34. Rodriguez JM, Reus M, Moreno A, Martinez M, Soria T, Carrasco $L$, et al. High-resolution ultrasound associated with aspiration biopsy in the follow-up of patients with differentiated thyroid cancer. Otolaryngol Head Neck Surg 1997;117:694-7.
35. Krishnamurthy S, Bedi DG, Caraway NP. Ultrasound-guided fine-needle aspiration biopsy of the thyroid bed. Cancer 2001;93:199-204.

36. Sclhumberger M, Baudin E. Serum thyroglobulin determination in the follow-up of patients with differentiated thyroid carcinoma. Eur J Endocrinol 1998;138:249-52.

37. Schlumberger M, Mancusi F, Baudin E, Pacini F. ${ }^{131}$ I therapy for elevated thyroglobulin levels. Thyroid 1997;7:273-6.

38. Maxon HR. Detection of residual and recurrent thyroid cancer by radionuclide imaging. Thyroid 1999;9:443-6.

39. Kessler A, Rappaport Y, Blank A, Marmor S, Weiss J, Graif M. Cystic appearance of cervical lymph nodes is characteristic of metastatic papillary thyroid carcinoma. J Clin Ultrasound 2003;31:21-5

\section{Endereço para correspondência:}

Rosa Paula M. Biscolla

Disciplina de Endocrinologia

Escola Paulista de Medicina/ Universidade Federal de São Paulo

Rua Pedro de Toledo 781, 120 andar

04039-032 São Paulo, SP

Fax: (1 1) 5084-5231

E-mail: rosapaula@uol.com.br 